\title{
Teaching how to expect the unexpected: improving the retention of knowledge for rare clinical events
}

\author{
M. Dylan Bould, MB · Viren N. Naik, MD
}

Published online: 17 December 2008

(C) Canadian Anesthesiologists' Society 2008

If you wish to forget anything on the spot, make a note that this thing is to be remembered

Edgar Allan Poe, 1844

Anesthesiologists must have a comprehensive understanding of many pathologies, technical pitfalls, and, most importantly, rarely seen but rapidly life threatening crisis situations. It is of little practical value to demonstrate knowledge immediately after learning about rare crises, as it is likely to be months, or even years, before that knowledge is required in clinical practice. Unfortunately, retaining skills and knowledge after being trained in uncommonly seen events is known to be problematic. Both staff anesthesiologists and residents have been found not to adhere to established guidelines during crisis management. ${ }^{1}$

In this issue of the Journal, Goldhaber-Fiebert et al. ${ }^{2}$ report on their investigation into the learning and retention of information following a 30-min lecture regarding seven rare but important medical errors. These investigators established objectives based on their departmental quality assurance database and adverse events reported by faculty, and they should be commended for incorporating the information into a formal residency curriculum in an effort to prevent future occurrences of critical incidents. The process of discussing reported critical incidents is valuable; furthermore, Goldhaber-Fiebert et al. seized the

M. D. Bould, MB (ه)

Department of Anesthesia, The Hospital for Sick Children, University of Toronto, 555 University Avenue, Toronto,

ON, Canada M5G 1X8

e-mail: dylan.bould@utoronto.ca

V. N. Naik, MD

Department of Anesthesia, St. Michael's Hospital, University of Toronto, Toronto, ON, Canada opportunity to use these crises and "near-misses" for a broader educational advantage.

Goldhaber-Fiebert et al. should also be congratulated for their attempts to measure the success of their lecture program. Instead of limiting themselves to the learners' subjective impressions of the teaching, they have examined its impact. Following testing with multiple-choice questions (MCQs) immediately after the lecture, they noted a $22.2 \%$ increase in the learners' knowledge of the material, compared to their assessment of the learners' knowledge of the same material prior to the lecture. In particular, their focus on retention of knowledge at 6 months is especially important. When the learners were retested at 6 months, their improvement from baseline fell to $7.9 \%$ compared to the control questions that were unrelated to the teaching intervention. To be precise, every other anesthesiologist at the lecture was simply answering one more of the seven questions correctly. Considering the clinical importance of the information presented in the lecture, we consider the next question to be: What could be done better?

In most institutions, lectures remain the most commonly used teaching modality in a formal medical education curriculum. As lectures appear to be in no immediate danger of extinction, it is essential that they be delivered effectively. There is the potential for effective and ineffective learning in all teaching methods. Important elements of good lecturing include clarifying the concepts, allowing students to be active learners, and demonstrating the processes of solving clinical problems. ${ }^{3}$ These elements are consistent with cognitive psychology research that demonstrates the importance of deep processing of information for its long-term retention. ${ }^{4}$ Varying the stimulus being delivered during a lecture is also important, as learning starts to decline almost immediately after lecturing begins. ${ }^{5}$ A detailed review of this subject is found 
elsewhere ${ }^{3}$ and ongoing quality assurance is warranted to investigate whether changes to lecturing style can improve retention of information.

Insights into improving the long-term retention of information from educational interventions can be gained from the basic cognitive psychological literature. The MCQ examination that followed the lecture in GoldhaberFiebert et al.'s study likely had a direct effect on the retention of that information. There is greatly improved knowledge retention from taking a test compared to simply rereading the material. The role of tests to improve learning is a generally under-appreciated concept in education that has recently been reviewed by Larsen et al. ${ }^{6}$ Although Goldhaber-Fiebert et al.'s study did not investigate this issue, it is interesting to speculate on the effect that the test given immediately after the lecture would have on the retention of information at 6 months. Tests that require learners to construct their own responses have a greater testing effect than MCQs, and perhaps a test based on short-answer questions would have resulted in different long-term retention. ${ }^{6}$ Finally, due to anonymization and the study design, feedback of the MCQ results to subjects was precluded to some extent; however, feedback enhances the testing effect, and there is some evidence that delayed feedback is more effective than immediate feedback on promoting long-term retention of information. ${ }^{7}$

Another well established influence on the retention of factual information is the duration of the learning sessions. Multiple short sessions are referred to as distributed learning, whereas one lengthy session is referred to as massed learning. Distributed learning, known as the spacing effect, results in superior long-term retention to massed learning. ${ }^{8}$ This suggests value in delivering short seminars as the basis for introducing information from the critical incident databases into a formal curriculum. Variations in the distribution of learning have the potential to be particularly effective for addressing uncommon clinical events. Different inter-study intervals (the time between teachings) result in better performance for different retention intervals (the time between the last episode of teaching and the time when retrieval of that information is required). Results from 1,800 subjects learning unfamiliar facts suggest that a 1-day inter-study interval is ideal for a 1-week retention interval, whereas the optimal inter-study interval for a retention interval of 1 year is 3 weeks. ${ }^{9}$

The principles of the testing effect, the spacing effect, and the effect of variations in feedback should be high on the agenda for medical education research. They offer many avenues for further research, as educational researchers are only beginning to investigate these psychological phenomena in practical educational settings. ${ }^{6}$

The investigators have demonstrated some retention of knowledge; however, how can we assess the meaningful translation of that learning into clinical practice? Miller's pyramid describes a hierarchy of evaluation beginning with the "knows" level, followed by the "knows how" level where knowledge is applied..$^{10}$ Both of these levels can be assessed using written examinations such as that used by Goldhaber-Fiebert et al. However, the "gold standard" of assessment should be whether learning impacts patient care, and this evaluation is best tested at the highest level of Miller's pyramid, i.e., the "does" level. Testing this level has rightly been described as "the international challenge of the century for all involved in clinical competencetesting". ${ }^{11}$ The fact that some clinical scenarios are a rarity renders them unlikely to be encountered during an individual's supervised training, and this, in turn, renders an individual unlikely to be assessed on the "does" level. On the way from "knows how" to "does" is "shows how", i.e., the demonstration of appropriate performance. For many clinical skills, "shows how" can be assessed using tools such as the Objective Structured Clinical Examinations and standardized patients. Simulation-based assessments for high-stakes purposes are controversial, ${ }^{12}$ but they are currently the only way to "show how" to manage rare clinical crises.

What should be the next steps after a study such as this? Goldhaber-Fiebert et al. state that their methodology could be used to compare different teaching modalities. A randomized controlled trial comparing their original lecture with an alternative technique could provide a robust methodology for investigating whether $7.9 \%$ retention can be improved. What alternatives should be considered for comparison? Simulation-based education employs the principle of deliberate practice, and this modality has demonstrated effective learning at 14 months with no significant decay in resuscitation skills and knowledge. ${ }^{13}$ This learning also appears to transfer to actual clinical practice. ${ }^{14}$ However, simulation is a time and resourceintensive teaching modality; therefore, it is not the most efficient and cost-effective method of delivering knowledge to large numbers of people in a timely fashion. Consequently, simulation should not be considered as a replacement for other modalities such as lectures, tutorials, problem-based learning, and e-learning. Rather, simulation should be viewed as complementary to other modalities, and any comparisons should reflect this important balance.

Due to their clinical significance, there are skills that all medical specialties must master to treat uncommon crises, despite their rarity. In anesthesiology and other acute care specialties, there may be insufficient time in a crisis situation to search for the relevant information or to seek advice from colleagues. The first step in crisis management education is to ensure that these skills are incorporated into a curriculum for both residency training and continuing medical education. The second step is to ensure effective 
teaching that promotes long-term retention, an aspect that is arguably neglected in medical education research. Finally, these skills must be evaluated in the contexts of research, residency training, and life-long learning, so that we can evaluate their impact on patient safety and, subsequently, direct further training.

\section{Enseigner La Façon De Prévoir l'imprévisible: l'amélioration De La Rétention Des Connaissances Portant Sur Les Événements Cliniques Rares}

«Le meilleur moyen d'oublier quelque chose immédiatement est de noter qu'on doit s'en souvenir. »

Edgar Allan Poe, 1844

Pour tout anesthésiologiste, il est essentiel de posséder une compréhension exhaustive de nombreuses pathologies, des écueils techniques potentiels, mais, plus important encore, de situations de crises survenant rarement mais devenant rapidement potentiellement fatales. La valeur pratique d'une démonstration des connaissances immédiatement après l'apprentissage entourant les situations de crise est futile, étant donné qu'il est plus probable que des mois, voire des années, passent avant que ces connaissances n'aient besoin d'être rappelées en mémoire pour être appliquées dans la pratique clinique. Malheureusement, il est reconnu que la rétention des compétences et des connaissances après une formation portant sur des événements rares est problématique. En effet, il a été observé que les anesthésiologistes aussi bien que les résidents ne respectaient pas les directives établies lors de la gestion de crises. ${ }^{1}$

Dans ce numéro du Journal, Goldhaber-Fiebert et coll. ${ }^{2}$ rapportent les résultats de leur enquête, qui s'intéressait à l'apprentissage et à la rétention des informations à la suite d'un cours de 30 min concernant sept erreurs médicales rares mais importantes. Ces chercheurs ont établi des objectifs se basant sur la banque de données en matière d'évaluation de la qualité de l'acte de leur département ainsi que sur les complications rapportées par les membres de la faculté, et leur effort d'intégration de ces informations dans un curriculum officiel pour les résidents afin d'éviter la survenue future d'incidents critiques doit être loué. D'une part, le processus qui consiste à analyser les incidents critiques survenus est important; d'autre part, Goldhaber-Fiebert et coll. profitent de l'occasion pour tirer parti de ces crises et celles évitées de justesse et les transformer en un avantage éducationnel plus large.
Il faut aussi féliciter Goldhaber-Fiebert et coll. pour leurs tentatives de mesures de la réussite de leur programme de cours. Au lieu de se limiter aux impressions subjectives des apprenants par rapport à la matière enseignée, les auteurs ont examiné son impact. À la suite d'un test à choix de réponses effectué immédiatement après le cours, les auteurs ont noté une augmentation de $22 \%$ du niveau de la connaissance de la matière des apprenants, par rapport à leur évaluation de leur connaissance de la même matière avant le cours. Plus particulièrement, leur concentration particulière sur la rétention des connaissances à six mois est d'une importance cruciale. Lorsque les apprenants ont à nouveau passé l'examen six mois plus tard, l'amélioration par rapport aux connaissances avant le cours a chuté à $7.9 \%$ comparativement aux questions témoins qui ne portaient pas sur la matière enseignée. Plus précisément, un anesthésiologiste sur deux ayant suivi le cours a répondu correctement à une question de plus sur les sept questions posées. Si l'on pense à l'importance clinique des informations présentées lors du cours, la prochaine question devrait être, selon nous: Que peut-on améliorer?

Dans la plupart des institutions, les cours demeurent la modalité d'enseignement la plus répandue dans un curriculum officiel de formation médicale. Étant donné que les cours ne sont pas en voie d'extinction, il est essentiel qu'ils soient donnés de façon efficace. Toute méthode d'enseignement porte en elle un potentiel d'apprentissage efficace ou inefficace. Parmi les éléments essentiels à un bon cours, il est important de clarifier les concepts, de permettre aux étudiants d'être des apprenants actifs, et de démontrer des procédés permettant de résoudre des problèmes cliniques. ${ }^{3}$ Ces éléments sont en accord avec les recherches menées en psychologie cognitive, lesquelles démontrent l'importance d'un traitement approfondi de l'information afin d'assurer sa rétention à long terme. ${ }^{4}$ La variation des stimuli proposés durant un cours est également importante, étant donné que l'apprentissage commence à décliner dès le tout début du cours. ${ }^{5}$ Un compte-rendu détaillé à ce sujet existe, ${ }^{3}$ et une évaluation de la qualité continue devrait être poursuivie pour déterminer si les changements apportés au style de cours peuvent améliorer la rétention de l'information.

Nous pouvons tirer certaines conclusions en étudiant la littérature en psychologie cognitive fondamentale concernant l'amélioration de la rétention à long terme des interventions éducationnelles. Dans l'étude de GoldhaberFiebert et coll., l'examen de questions à choix de réponses qui a suivi le cours a probablement eu un effet direct sur la rétention de ces informations. En effet, la rétention des connaissances est bien plus forte lorsque les apprenants doivent passer un test, comparativement à une simple relecture de la matière. Le rôle des tests dans l'amélioration de l'apprentissage est un concept communément sousapprécié dans l'éducation, comme l'ont récemment 
rapporté Larsen et coll. ${ }^{6}$ Bien que l'étude de GoldhaberFiebert et coll. n'ait pas porté sur cette question, il est intéressant de se poser la question de l'effet que le test donné immédiatement après le cours aurait eu sur la rétention des informations à six mois. Les tests qui nécessitent des apprenants qu'ils élaborent leurs propres réponses ont un impact de test plus important que les questions à choix de réponses, et il est possible qu'un test basé sur des questions à réponses courtes aurait eu un impact différent sur la rétention à long terme. ${ }^{6}$ Finalement, en raison de l'anonymisation et du concept de l'étude, la rétroaction aux sujets de l'étude des résultats du test à choix de réponses a été quelque peu limitée; pourtant, la rétroaction améliore l'effet de test, et nous avons des données probantes qu'une rétroaction retardée est plus efficace qu'une rétroaction immédiate pour promouvoir la rétention d'informations à long terme. ${ }^{7}$

La durée des sessions d'apprentissage est un autre facteur bien connu qui influence la rétention d'informations factuelles. Lorsqu'il s'agit de multiples sessions brèves, on parlera d'apprentissage distribué, alors que pour une longue session, le terme employé est apprentissage massé. L'apprentissage distribué, connu sous le nom d'effet d'espacement, engendre une meilleure rétention à long terme que l'apprentissage massé. ${ }^{8}$ Ainsi, il serait plus utile de proposer des cours brefs pour présenter les informations tirées des bases de données d'événements critiques dans un curriculum officiel. Les variations dans la distribution de l'apprentissage pourraient être particulièrement efficaces lors de la présentation d'événements cliniques peu courants. Des intervalles variés entre les sessions d'étude, ou le temps entre les apprentissages, donnent de meilleurs résultats en termes de performances pour différents intervalles de rétention, c'est-à-dire le temps entre la dernière session d'apprentissage et le moment où la remémoration de ces informations est nécessaire. Les résultats d'une étude portant sur 1800 sujets apprenant des faits inconnus suggèrent qu'un intervalle d'un jour entre les sessions d'étude est idéal pour un intervalle de rétention d'une semaine, alors que l'intervalle entre les sessions d'étude idéal pour un intervalle de rétention d'une année est de trois semaines. ${ }^{9}$

Les principes de l'effet de test, de l'effet d'espacement et de l'effet des variations de rétroaction devraient figurer parmi les priorités de la recherche en éducation médicale. En effet, ils offrent de nombreuses pistes à explorer dans les recherches futures, étant donné que les chercheurs en éducation commencent à explorer ces phénomènes psychologiques dans des contextes d'éducation pratique. ${ }^{6}$

Goldhaber-Fiebert et coll. ont démontré qu'il y avait une certaine rétention des connaissances; toutefois, est-il possible d'évaluer l'application de cet apprentissage dans la clinique pratique ? La pyramide de Miller décrit une hiérarchie d'évaluation, qui commence par le niveau du «savoir », puis est suivi par le niveau du «savoir comment», dans lequel les connaissances sont mises en pratique. ${ }^{10}$ Ces deux niveaux peuvent être évalués à l'aide d'examens écrits tels que ceux utilisés par GoldhaberFiebert et coll. Cependant, l'étalon or de l'évaluation devrait porter sur l'impact de cet apprentissage sur les soins aux patients, et le meilleur endroit pour tester cet impact est situé au niveau le plus élevé de la pyramide de Miller, c'est-à-dire, au niveau du «faire ». L'évaluation de ce niveau a été décrite, à juste titre, comme «le défi international du siècle pour toute personne impliquée dans l'évaluation des compétences cliniques $\gg .{ }^{11}$ Le fait que les scénarios cliniques soient rares a pour résultat qu'il est peu probable que de tels scénarios soient utilisés dans la formation supervisée d'une personne, ce qui, par la suite, a pour résultat qu'il est peu probable qu'une personne soit évaluée au niveau du «faire ». Il y a un quatrième niveau dans la pyramide de Miller, situé entre le «savoir comment » et le «faire » : le «montrer comment », c'est-àdire la démonstration d'une performance adéquate. Dans le cas de nombreuses compétences cliniques, le «montrer comment » peut être évalué à l'aide d'outils tels que les examens cliniques par objectifs structurés et les patients simulés. Les évaluations basées sur des simulations pour des objectifs à grands enjeux sont controversées, ${ }^{12}$ mais constituent le seul moyen à l'heure actuelle de «montrer comment » prendre en charge des crises cliniques rares.

Après une étude telle que celle de Goldhaber-Fiebert et coll., quelles sont les prochaines étapes? Les auteurs affirment que leur méthodologie pourrait servir à comparer différentes modalités d'enseignement. Une étude randomisée contrôlée comparant leur cours original à une autre technique pourrait offrir une méthodologie solide qui permettrait d'évaluer si le taux de rétention de $7.9 \%$ peut être amélioré. Quelles alternatives prendre en compte pour réaliser une comparaison ? L'éducation basée sur les simulations se fonde sur le principe de la pratique délibérée, et cette modalité s'est avérée être un outil d'apprentissage efficace à 14 mois, ne montrant pas de déclin significatif des compétences et connaissances en matière de réanimation. ${ }^{13}$ Ce type d'apprentissage semble également se traduire dans la pratique clinique. ${ }^{14}$ Cependant, la simulation est une modalité d'enseignement qui exige beaucoup de temps et de ressources; pour cette raison, il ne s'agit pas de la méthode la plus efficace et la plus rentable pour la transmission des connaissances à un grand nombre de personnes en temps opportun. Dès lors, la simulation ne devrait pas être considérée comme une méthode pouvant remplacer d'autres modalités d'enseignement comme les cours, les enseignements individuels, l'apprentissage par problème et l'apprentissage sur Internet. La simulation devrait plutôt être considérée comme un 
complément à d'autres modalités, et toute comparaison devrait refléter cet équilibre important.

En raison de leur importance clinique, certaines compétences doivent être maîtrisées par toutes les spécialités médicales afin de prendre en charge les crises peu courantes et ce, malgré leur rareté. En anesthésiologie et dans d'autres spécialités des soins aigus, il se peut qu'il n'y ait pas suffisamment de temps dans une situation de crise pour chercher les informations pertinentes ou pour demander l'avis d'un collègue. La première étape de l'éducation en matière de gestion de crise est de s'assurer que ces compétences sont intégrées dans un curriculum de formation en résidence et de formation médicale continue. La deuxième étape est d'assurer un enseignement efficace qui favorise la rétention des connaissances à long terme, un aspect quelque peu négligé dans la recherche en éducation médicale. Enfin, ces compétences doivent être évaluées dans les contextes de la recherche, de la formation en résidence, et de l'apprentissage permanent, de telle manière à pouvoir évaluer leur impact sur la sécurité des patients et, par la suite, orienter la formation future.

Conflicts of interest None declared.

\section{References}

1. Kurrek MM, Devitt JH, Cohen M. Cardiac arrest in the OR: how are our ACLS skills? Can J Anaesth 1998; 45: 130-2.
2. Goldhaber-Fiebert SN, Goldhaber-Fiebert JD, Rosow CE. Knowledge-based errors in anesthesia: a paired, controlled trial of learning and retention. Can J Anesth 2009; 56: 1.

3. Brown G, Manogue M. AMEE medical education guide no. 22: refreshing lecturing: a guide for lecturers. Med Teach 2001; 23: 231-44.

4. Roediger HL, Gallo DA, Geraci L. Processing approaches to cognition. In: Conway MA, editor. Levels of Processing 30 Years: On a Special Issue of Memory. Hove and New York: Psychology Press; 2002. p. 345-8.

5. Bligh DA. What's the Use of Lectures?. San Francisco: JosseyBass Publishers; 2000.

6. Larsen DP, Butler AC, Roediger HL III. Test-enhanced learning in medical education. Med Educ 2008; 42: 959-66.

7. Butler AC, Karpicke JD, Roediger HL III. The effect of type and timing of feedback on learning from multiple-choice tests. J Exp Psychol Appl 2007; 13: 273-81.

8. Cepeda NJ, Pashler H, Vul E, Wixted JT, Rohrer D. Distributed practice in verbal recall tasks: a review and quantitative synthesis. Psychol Bull 2006; 132: 354-80.

9. Pashler H, Rohrer D, Cepeda NJ, Carpenter SK. Enhancing learning and retarding forgetting: choices and consequences. Psychon Bull Rev 2007; 14: 187-93.

10. Miller GE. The assessment of clinical skills/competence/performance. Acad Med 1990; 65: S63-7.

11. Wass V, Van der Vleuten C, Shatzer J, Jones R. Assessment of clinical competence. Lancet 2001; 357: 945-9.

12. Savoldelli GL, Naik VN, Joo HS, et al. Evaluation of patient simulator performance as an adjunct to the oral examination for senior anesthesia residents. Anesthesiology 2006; 104: 475-81.

13. Wayne DB, Siddall VJ, Butter J, et al. A longitudinal study of internal medicine residents' retention of advanced cardiac life support skills. Acad Med 2006; 81: S9-12.

14. Wayne DB, Didwania A, Feinglass J, Fudala MJ, Barsuk JH, McGaghie WC. Simulation-based education improves quality of care during cardiac arrest team responses at an academic teaching hospital: a case-control study. Chest 2008; 133: 56-61. 